dessen «Untergründe» freilich noch kaum erahnen lassen, ob seine Länder einer positiven oder negativen Transformation ihrer Kulturlandschaft entgegengehen werden.

\author{
DE NEW YORK A PANAMA PAR LA TERRE FERME IL Y A 25 ANS
}

Il y a 25 ans un voyage de New York à Panama par la terre ferme offrait beaucoup d'autres aspects qu'aujourd'hui. L'achèvement du «Panamerican Highway» a amélioré considérablement les voies de communication en Amérique Centrale. Après cette période nous constatons aussi par parties des changements importants dans le paysage culturel, ce qui est bien visible par le redoublement de la population (de 23 à 46 millions d'habitants). Nonobstant nous rencontrons encore toujours le long de cette route internationale les contrastes des régions densement peuplées, cultivées et d'un fort développement économique et des paysages d'une nature intouchée, bien qu'il y aît eu des progrès de différentes manières dans les états de l'Amérique Centrale.

\title{
DER BEGRIFF «TRANSHUMANZ» IM ENGLISCHEN SPRACHGEBIET
}

\author{
ROLF GUBLER
}

Im englischen Sprachgebiet erfreut sich der Begriff «Transhumanz» einer relativ großen Popularität. Dazu trägt allerdings die Tatsache viel bei, daß man darunter eine etwas andere Vorstellung hat als etwa im Bereich der romanischen Sprachen oder des Deutschen. So findet man z. B.im «German and English Glossary of Geographical Terms» (1 [siehe Anmerkungen]) : Transhumanz = Almwanderung. King (2) setzt Transhumanz mit Wanderung von Volksgruppen (Transhumance, or migration of population groups...) gleich und schreibt auch, daß perennierende Transhumanz (perennial transhumance) äquivalent zu Nomadismus sei. Gottmann (3) versteht unter Transhumanz in Frankreich die Wanderungen von Großvieh und Schafen, welche von Ställen oder Tieflandweiden aus auf die Alpweiden führen. In «Elements of Geography» (4) wird Transhumanz als eine besondere Form von «livestock ranching» charakterisiert, bei welcher die Sommerweiden gepachtet werden. Hoffmann (5) versteht unter Transhumanz Senntenwirtschaft oder Sennerei. Beinahe dieselbe unpräzise Definition wie Newbegin (6) (Transhumanz is the periodic and alternating displacement of flocks and herds between two regions of different climate) gibt Stamp (7), der unter Transhumanz Wanderungen zwischen Regionen verschiedener Klimate versteht. Ähnlich äußert sich auch James (8). Im Oxford Wörterbuch (9) versteht man noch allgemeiner einfach eine jahreszeitliche Wanderung von Vieh zu einer andern Region. Moore (10) präzisiert, daß bei Transhumanz die Herden von Hirten, manchmal auch von erheblichen Bevölkerungsteilen begleitet werden und daß diese sowohl in den Bergen als auch im Tal Dauersiedlungen bewohnen.

Auch in Spezialarbeiten wird unter Transhumanz oft eine Art von Viehhaltung verstanden, die der Alpwirtschaft oder Säterwirtschaft entspricht (11). Dazu gehört z. B. die Arbeit über Transhumanz in Nord Skye von MacSween (12). Ebenso häufig aber lassen sich aus den gegebenen Unterlagen nicht klar die Schlüsse ziehen, um was für eine Art der Viehhaltung es sich handelt, da über die wesentlichen Punkte hinweggegangen wird. Dies dürfte im Fall der Transhumanz in «Tasmanien» (13) zutreffen.

Stellt man die im englischen Sprachbereich üblichen Definitionen und Beschreibungen von Transhumanz den nicht-englischen (14) gegenüber, so ergeben sich ganz wesentliche Unterschiede. Im besondern versteht man unter Transhumanz im englischen Sprachbereich auch Wirtschaftsformen der Viehhaltung, die sonst ausdrücklich nicht 
als Transhumanz bezeichnet werden, so z. B. die Alpwirtschaft (15). Es wäre deshalb sehr zu begrüßen, wenn diese erweiterte, unpräzise und nur Unklarheit schaffende englische Anwendung des Begriffs Transhumanz wieder fallen gelassen würde.

1 Fischer, Eric und Elliot: A German and English Glossary of Geographical Terms. American Geographical Society, 1950. 2 King, H. W.: The Pattern of Human Activities. Sydney 1949, XX, p.105. 3 Gottmann, Jean: A Geography of Europe. New York 1950, p.105. 4 Finch,Trewartha, Robinson, Hammond: Elements of Geography. New York 1957, p. 579. 5 Hoffmann, G.W.: A Geography of Europe. London 1961, p. 393. 6 Newbegin, M. I.: Modern Geography. 1911, p. 179. 7 Stamp, L. D.: Geographical terms. London 1961, p. 458 . 8 James, P. E.: An Outline of Geography, Boston 1935, p. 33of. 9 The Concise Oxford Dictionary. Oxford 1961. 1o Moore, W. G.: A Dictionary of Geography. Penguin Books, Harmondsworth, 1959. 11 Darum versucht auch B. Hofmeister in seiner Transhumanzbibliographie solche Arbeiten auszuscheiden (in Erdkunde, Band XV, 1961, p. 132). 12 The Scottish Geographical Magazine, Vol. 75, Sept. 1959, p. 75 ff. 13 Scott, Peter: Transhumance in Tasmania. New Zealand Geographer, Vol. XI, October 1955, p. $155 \mathrm{ff} .14$ Siehe Zusammenstellung davon von R. Gubler: Moderne Transhumanz in der Schweiz. Winterthur 1962, p. 2 ff. 15 Wenn Hofmeister (The Professional Geographer, Vol. XIII, March 1961) von einer allgemeinen Übereinkunft — «general agreement» - über den Begriff Transhumanz schreibt, so kann sich dies nur um die nicht englischsprachigen Autoren handeln.

\section{SUMMARY}

The term «transhumance», originating in Spain, France and Italy, has quite a clear and precise meaning on the Continent. The definition and use of this term in English publications is much more general and includes different kinds of animal husbandry which are explicitly not called «transhumance» on the Continent. It is therefore suggested that the English speaking authors, too, would use the term in the restrictive continental sense.

\section{GESELLSCHAFTSTÄTIGKEIT - ACTIVITÉ DES SOCIÉTÉS}

Fédération des sociétés suisses de géographie. Assemblée de la SHSN.

La prochaine assemblée des délégués de la Fédération des sociétés suisses de géographie aura lieu à Zurich, le samedi 1o octobre 1964, à l'occasion de la rencontre de la Société helvétique des sciences naturelles $(9,10,11$ octobre).

Local de la séance: Institut de géographie de l'EPF. Heure de la séance: au début de l'aprèsmidi du 1o octobre. Ordre du jour: Procès-verbal de la dernière séance. Rapports de caisse et des vérificateurs de comptes. Commission de recherche. «Nouvelles sections»de géographie en dehors des grandes villes suisses. Divers.

Programme prévu pour les $9,10,11$ octobre: Le 9, dans l'après-midi: conférences générales. Le 1o, au matin: communications. Le 1o, dans l'après-midi: Assemblée des délégués, puis excursion. Le déjeuner aura lieu à $Z$ urich, le dîner à Kloten. Le 11, ad libitum.

Conférences: Comme l'indique le procès-verbal ci-joint, les présidents des sociétés voudront bien avertir les conférenciers de m'envoyer le titre de leur communication et les indications concernant les clichés avant le 20 juin. Les communications ne doivent pas dépasser 15 minutes, afin de ménager quelques instants de discussion. En outre, je prie les conférenciers de préparer pour le jour de la rencontre un résumé de leur communication (une page imprimée au maximum). Le président central: E. L. Paillard, Avenue Jomini 1, Lausanne

Geographisch-Ethnographische Gesellschaft Zürich. Jahresbericht 1963/64.

Unsere Gesellschaft zählt gegenwärtig 456 Mitglieder, das sind $10 \mathrm{mehr}$ als vor Jahresfrist. 8 Mitglieder sind im Berichtsjahr verstorben, nämlich die Herren Dr. H. Freysz, Zürich, 37 Jahre Mitglied; Hch. Hürlimann, Küsnacht, 45 Jahre Mitglied; Hans Mahler, Küsnacht, 32 Jahre Mitglied; Dr. E. Guggenheim, Küsnacht, 21 Jahre Mitglied; Emil Meierhans, Zürich, 1 Jahr Mit- 